\title{
Validation of the effectiveness of ultrasonography as a diagnostic method for temporomandibular joint disorders and a comparison with MRI and CBCT
}

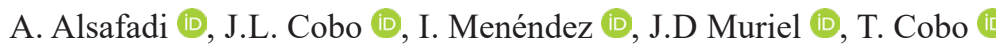

\begin{abstract}
Objective: The objective of this study was to compare findings from ultrasonography imaging (USI) of the temporomandibular joint (TMJ) with those from magnetic resonance imaging (MRI) and cone-beam computerized tomography (CBCT).

Methods: A total of 102 patients were included in this study. USI, MRI, and CBCT were performed in the TMJ area for all patients.

Results: USI showed $100 \%$ sensitivity (Se), $82.76 \%$ specificity (Sp), 93.15\% positive predictive value (PPV), 100\% negative predictive value (NPV) and $94.85 \%$ accuracy relative to MRI for identifying anterior disc displacement (ADD), while the Se, Sp, PPV, NPV, and accuracy were $100 \%$ for identifying joint effusion, relative to MRI. Moreover, USI showed a high agreement with CBCT, which had 98.08\% Se, 94\% Sp, 94.44\% PPV, 97.92\% NPV and $96.08 \%$ accuracy for identifying condylar irregularities, while MRI showed a $100 \%$ Se, $56.86 \%$ Sp, $69.86 \%$ PPV, $100 \%$ $\mathrm{NPV}$, and $78.43 \%$ accuracy for detecting condylar irregularities, relative to $\mathrm{CBCT}$.

Conclusions: High-resolution USI is a useful diagnostic method for detecting TMJ pathologies; USI can supplement clinical evaluations for patients with temporomandibular joint disorders (TMDs), and this imaging modality can be used as a diagnostic tool to identify internal derangement of the TMJ.

Keywords: Temporomandibular joint, Ultrasonography, Articular disc, Disc displacement, Effusion, Condylar irregularity
\end{abstract}

Alaa Alsafadi (®)

alaa.alsafadi@hotmail.com

A. Alsafadi, I. Menéndez, T. Cobo

Department of Surgery and Medical-Surgical Specialties, University of Oviedo, Spain

Instituto Asturiano de Odontología

J.L. Cobo

Oral and Maxillofacial Surgery Service, Central University Hospital of Asturias, Oviedo, Spain

J.D Muriel

Image center, Instituto Asturiano de Odontología. Oviedo, Spain

Submitted / Gönderilme: 29.05.2019

Accepted / Kabul: 08.10.2019

\section{Introduction}

The human temporomandibular joint (TMJ) is a diarthrodial joint; each joint consists of the mandibular condyle and its corresponding temporal cavity (articular eminence and glenoid fossa). The TMJ and its associated structures play an essential role in guiding mandibular movements and distributing the stresses produced by chewing, speaking and swallowing (1).

Temporomandibular joint disorders (TMDs) are a common source of orofacial pain according to the World Health Organization (WHO). TMDs are characterized by limited mouth opening, are accompanied by a clicking noise, and affect the masticatory system and its associated structures $(2,3)$. These disorders affect approximately 10 $70 \%$ of the population (4). TMD disorders are classified as muscular diseases, internal derangement (ID), inflammatory disease and osteoarthritis (3). The need for imaging to diagnosis TMD is still controversial; therefore, according to some investigators, practitioners should perform imaging only in cases with reasonable exceptions where imaging data would add additional information for the diagnosis (5). In contrast, other investigators reported that TMJ abnormalities could not be assessed by only clinical examinations, and an essential aspect in the diagnosis of TMD is the clinical examination followed by an imaging technique to support and confirm the diagnosis, while considering the chief patient complaints, clinical history, clinical findings, radiation dose of the imaging technique and examination costs (1). Several studies have discussed for the use, accuracy, strengths, and limitations of various imaging techniques as diagnostic methods. Magnetic resonance imaging (MRI) is considered the gold standard modality due to its efficacy in evaluating the soft and hard tissues of the TMJ (5). However, MRIs are high-cost 
examinations due to the need for expensive equipment. Recently, high-resolution ultrasonography (USI), which is considered a non-invasive method for the diagnosis of TMD, has been shown to be a more consistent and lower cost technique than other technologies. The main advantage of USI is the ability to perform static and dynamic studies; this allows the practitioner to detect the disc position more accurately than with a single static investigation. USI can not only demonstrate soft tissue alterations but also visualize hard tissue alterations (2).

Furthermore, USI shows an agreement with MRI in detecting normal condyle disc anatomy, anterior disc displacement (ADD) without reduction, ADD with reduction, joint effusion and condylar irregularity. The diagnostic accuracy of USI for detecting disc displacement is $79.3 \%$, and its sensitivity (Se) and specificity ( $\mathrm{Sp}$ ) have been reported as $72.7 \%$ and $77.5 \%$, respectively (6). However, the Se and Sp of USI have been found to be higher in studies with more powerful probes ( $12 \mathrm{MHz}$ ), thus providing better tissue differentiation(3). USI is based on ultrasound waves, which are emitted by a transducer, go through soft and hard tissues and are partially reflected by different densities while transiting through different anatomical structures. These sound waves are reflected again through the emitting probe and translated into images (7). The purpose of this paper is to evaluate the diagnostic efficacy of USI in detecting TMJ dysfunction and to compare USI with MRI in detecting anterior disc displacement with reduction (ADDwr), anterior disc displacement without reduction (ADDwor), and joint effusion. The second approach compared the efficacy of USI, MRI, and cone-beam computed tomography (CBCT) in detecting condylar irregularities, while bearing in mind that $\mathrm{CBCT}$ is the gold standard for detecting any structural bone changes.

\section{Materials and methods}

The study evaluated one hundred and two (102) patients and 204 joints, grouped in 25 men $(24.5 \%)$ and 77 women (75.5\%); the mean age of the patients was 31.4 years, and the median was 32 years old. These patients came to our clinic between September 2016 and November 2018 with pain in the TMJ area. The patients were in healthy condition; they did not receive any treatments or have any related critical diseases. The exclusion criteria of this study included previous trauma to the head and face area, prior surgery on the TMJ, orthodontic treatment, and facial deformities. Extraoral and intraoral exploration and clinical assessments of the TMJ were conducted by the same practitioner (orthodontist) per the research diagnostic criteria for temporomandibular disorders (CDC/TMD) (8). The examination included evaluating the patient history, assessing the presence or absence of joint sound and pain, palpating the intraoral and extraoral masticatory muscles, and measuring the mouth opening range. A clinical examination was carried out for all patients, followed by USI, MRI and CBCT examinations. The patients were not allowed to receive any treatment between surveys. Written informed consent was acquired from each subject, and all participants were informed about the study's objective and procedures. Ethical approval was obtained from the ethics committee of the Instituto Asturiano de Odontologia (IAO) (Ref. IAO-16-060), and this study met the criteria of Helsinki 1975, 2008 revision and Spanish legalization.

\section{Clinical evaluation and diagnostic imaging}

Three imaging methods were performed for 102 patients. For each patient, two ultrasonographic examinations, including static and dynamic studies, were performed on both condyles. A dynamic analysis was performed with the patient continuously closing and opening the mouth to exclude disc displacement (9). The three imaging scans were interpreted by the same researchers (an orthodontist and maxillofacial surgeon experienced in TMDs). When joint disorders (disc displacement, joint effusion, and condylar irregularities) were detected, we classified the disorders as "unilateral" or "bilateral"; in cases of no joint disorders, we classified the findings as "normal." The mean examination times for USI, MRI and CBCT were 10, 20 and 4 minutes, respectively.

\section{Ultrasonography}

B-mode ultrasonographic examinations were carried out with a LOGIQ e ultrasound (BT12-GE Healthcare, USA) with an $18 \mathrm{MHz}$ high-frequency transducer. The transducer used in this study for musculoskeletal imaging purposes was an L8-18i wideband high-frequency "hockey stick" linear array, which has a 6.7-18.0 MHz imaging frequency. The patient was imaged under appropriate lighting in a dental chair while in a supine position. The probe was positioned over the TMJ area perpendicular to the zygomatic arch in the transverse and longitudinal planes and tilted until the best visualization was achieved (4,5,9-12) (Figure 1). 


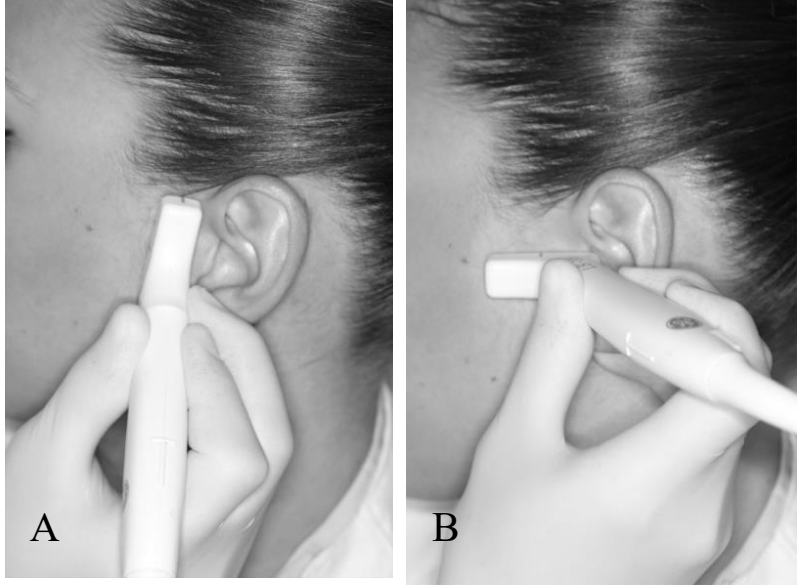

Figure 1. A. USI probe placed perpendicular to the zygomatic arch (transverse). B. USI probe placed parallel to the zygomatic arch (sagittal). Both techniques are applied in the closed and open mouth positions.

The images were captured in both the closed and maximal mouth opening positions. On the ultrasound images, the condylar surface and articular eminence appeared as hyperechogenic lines, while the articular disc was identified by a thin hypoechogenic band between the two lines. The relationship between the articular disc and the condyle was assessed. The disc position was classified as a normal position when the anterior border of the disc was located superior to the condyle and as anteriorly displaced when the anterior border of the disc was presented anterior to the condyle. Furthermore, joint effusion was identified by indirect measurements of capsular distension. This measurement was made between the condylar lateralsuperior surface and the articular capsule with the patient in the closed-mouth position .

\section{Magnetic resonance imaging}

MRI was performed after the USI examination to assess if the results obtained by USI coincided with those obtained by MRI. The MRI examination was carried out by a Signa pioneer GE Healthcare $3.0 \mathrm{~T} 70 \mathrm{~cm}$. A sagittal study of the TMJ area was performed with the following MRI protocol:

The patient placed on the exploratory table in the supine position, and dual-surface coils were placed on the patient on both joints, focusing on the anterior part of the talus of the ears so that the images could be acquired simultaneously. An axial image (locator) was acquired with the mouth closed to enable the programme to place precise perpendicular cuts to the condyle to acquire images in the sagittal plane. Then, precise parallel cuts to the condyle were placed in the programme to obtain images in the coronal plane. Next, the patient was told to open his or her mouth as much as possible, and lingual depressors were used to keep the mouth open; the axial locator scan was repeated, and the programme again positioned different cuts in the same planes as with the mouth closed. On MRI, disc positions were categorized as normal or displaced according to previous criteria.

\section{Cone-beam computed tomography}

CBCT examinations were carried out with a Planmeca ProMax 3D Mid device (Helsinki - Finland, 2015) with an image pixel resolution of $17.1 \times 8.0 \mathrm{~cm}$, volumetric pixel of $200 \mathrm{~nm}, 90 \mathrm{kV}, 8.0 \mathrm{~mA}$, and a scan time of 12.312 seconds with Romexis 5.2.1.R of software. CBCT was performed to confirm the presence of condylar irregularities that were observed with USI. The images were taken with the patient in a standing position, and the head was parallel to the Frankfurt plane.

\section{Statistical analysis}

A descriptive analysis of each variable was performed, which provided the absolute and relative distribution for the qualitative variables as well as the position measurements such as the mean, median, minimum, and maximum, or dispersion measures such as the standard deviation for quantitative variables. The relationships between qualitative variables were assessed with Pearson's chi-square or Fisher's tests, depending on the hypothesis about expected frequencies. Values such as Sensitivity (Se), Specificity $(\mathrm{Sp})$, positive predictive value (PPV), negative predictive value (NPV) and Accuracy were calculated to evaluate the diagnostic quality of the imaging modalities used to detect the studied pathologies. The level of significance was set at 0.05 , and all statistical analyses were performed using R Statistical Software (version 3.4.4; R Foundation for Statistical Computing, Vienna, Austria).

\section{Result}

In the present study, the articular disc was identified in all joints (204) by ultrasonography in both the closed and opened mouth positions. One hundred and two patients, including 25 males (24.5\%) and 77 females (75.5\%) with a mean age of 31.4 years, were evaluated with three different imaging modalities: USI, MRI and CBCT. 
USI identified the following pathologies related to the TMJ: ADDwr, ADDwor, joint effusion, and condylar irregularity. USI showed that 29 (28.4\%) patients had a normal disc position (Figure 2), 39 (38.2\%) patients had bilateral ADDwr, 30 (29.4\%) patients had unilateral ADDwr, and 4 (3.9\%) patients had ADDwor (Figure 3).
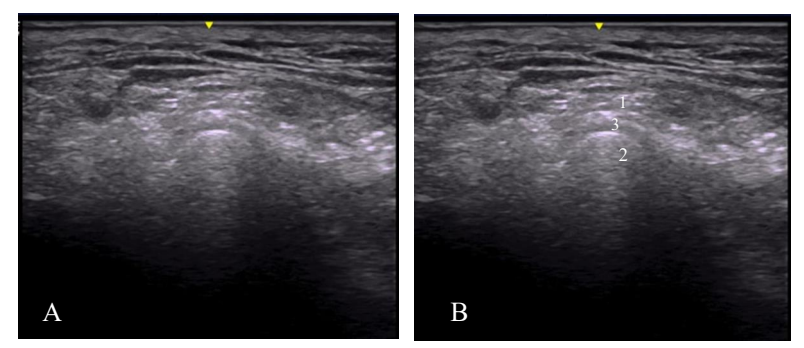

Figure 2. A. Normal position of the articular disc on a sonogram. B. The same sonogram where the anatomical structures are identified by numbers. 1 - Capsule, 2 - Mandibular condyle, 3 - Articular disc, 4 - Articular eminence.
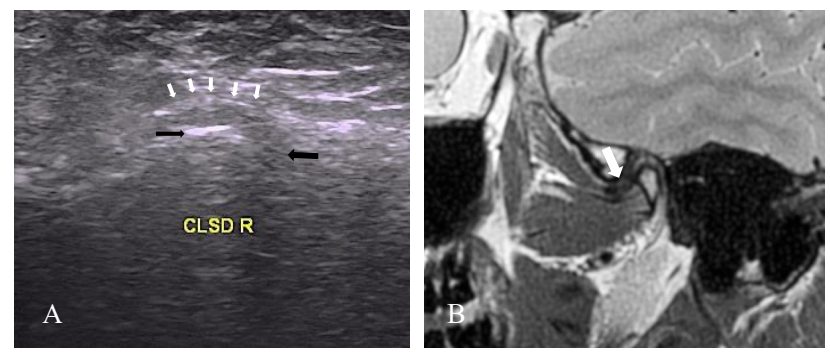

Figure 3. Ultrasonography and MRI for the same patient. A. USI showing anterior disc displacement without reduction. The small white arrows indicate the glenoid fossa, and the thin black arrow indicates the condyle while the thick black arrow indicates the articular disc position. B. MRI showing agreement with USI, in which the articular disc is displaced anteriorly without reduction; the white arrow indicates the articular disc position.

However, no joint effusion "normal" was also seen in 49 (48.0\%) patients by USI, while unilateral joint effusion was identified in 21 (20.6\%) patients, and bilateral joint effusion was identified in $32(31.4 \%)$ patients, (Figure 4.A).
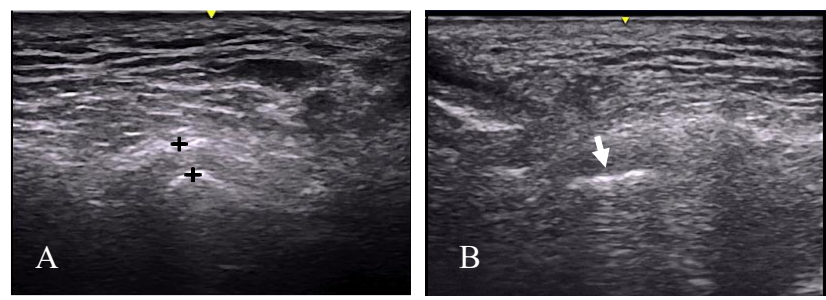

Figure 4. A. Joint effusion on the left side of the mandible. This sonogram was taken in the closed mouth position. B. Condylar flattening with joint effusion on the right side of the mandible, with the patient in the closed mouth position.
Condylar irregularities were clearly seen on the sonogram, as in (Figure 4.B). Normal condyle morphology was observed in $51(50.0 \%)$ patients, whereas unilateral condylar irregularities were present in $30(29.4 \%)$ patients, and bilateral condylar irregularities were present in 21 (20.6\%) patients. Fisher's test showed a statistically significant relationship between sex and disc displacement $(p<0.001)$, and Pearson's chi-squared test showed a statistically significant relationship between sex, joint effusion and condylar irregularities $(p<0.001)$.

Furthermore, MRI examination results concerning disc position are as follows: 34 (33.3\%) patients with normal disc position, $20(19.6 \%)$ patients with unilateral ADDwr, 44 (43.1\%) patients with bilateral ADDwr and 4 (3.9\%) patients with ADDwor. In terms of joint effusion, MRI showed that $49(48.0 \%)$ patients had normal joint spacing, while $26(25.5 \%)$ patients had unilateral joint effusion, and $27(26.5 \%)$ patients had bilateral joint effusion. Concerning condylar irregularity, MRI diagnosed 49 (48.0\%) patients with healthy condyles, 33 (32.4\%) patients with unilateral condylar irregularities and $20(19.6 \%)$ patients as bilateral condylar irregularities.

However, Pearson's chi-squared test showed a statistically significant relationship between sex and joint effusion detected by MRI $(p<0.001)$, while Fisher's test showed a statistically significant relationship between sex and disc displacement $(p<0.001)$ and a nonsignificant relationship between sex and condylar irregularities $(p=$ 0.074).

CBCT was performed for the same sample; Pearson's chi-square test showed a statistically significant relationship between sex and condylar irregularities $(p<0.001)$. CBCT show $51(50 \%)$ patients presents normal condyles, while $21(20.5 \%)$ patients present bilateral condylar irregularities and $30(29.4 \%)$ patients presents unilateral condylar irregularities. However, USI detects 48 patients (47\%) with normal condyles, 33 patients (32.3\%) with bilateral condyle irregularity and 21(20.5\%) patients with unilateral condyle irregularity, while MRI detects 29 patients (28.4\%) with normal condyles, 48 patients (47\%) with unilateral condyle irregularity, and 25 patients (24.5\%) with bilateral condyle irregularity. Figure 5 demonstrates representative images acquired by the three imaging modalities (USI, MRI, and CBCT) for the same patient. USI and MRI detected partial ADDwr of the articular disc, whereas USI and CBCT detected condylar flattening and asymmetric morphology. 


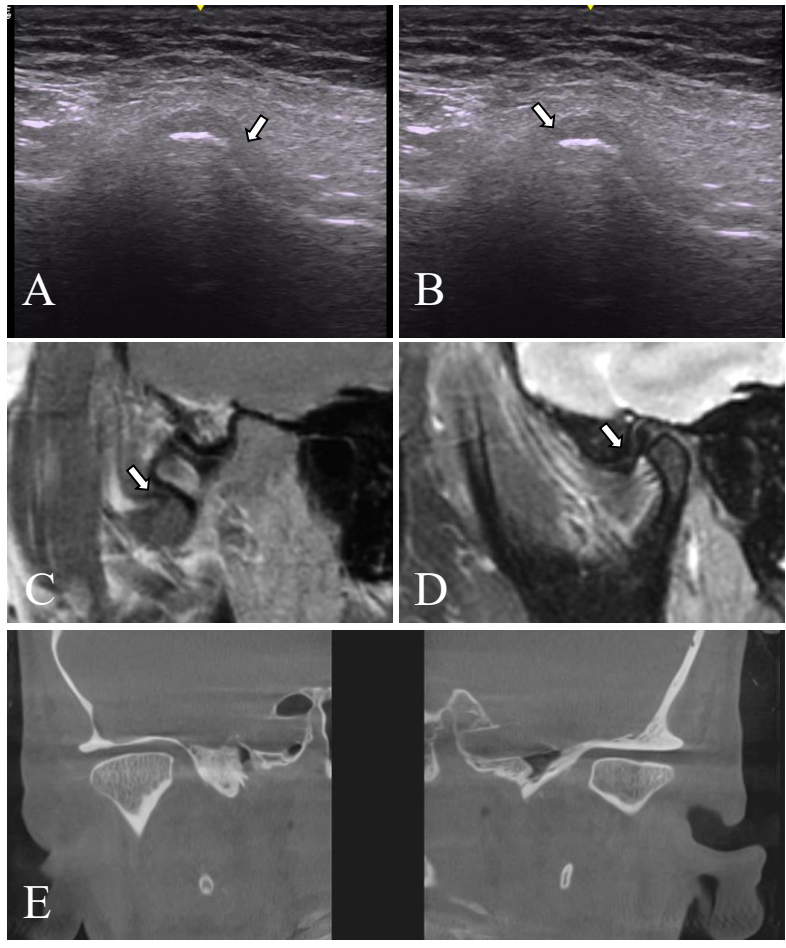

Figure 5. Images from the three imaging modalities (USI, MRI, and CBCT) for the same patient. A \& B. Sagittal USI of the right and left side of the mandible showing ADDwr; the arrow indicates the articular disc position. C \& D Sagittal MRI of the right and left side of the mandible, which is in agreement with the USI results for the articular disc position. E. Axial CBCT of the TMJ showing flattening and asymmetric al morphology of the condyle on the left side of the mandible, which is compatible with the results from USI.

To evaluate the use of USI as a diagnostic method, the Sp, Se, PPV, NPV and accuracy were calculated for the ability of both MRI and USI to detect disc displacement, joint effusion, and condylar irregularity. Additionally, a diagnostic test was performed to evaluate the three imaging modalities, MRI, USI, and CBCT, in terms of detecting condylar irregularities. The detection of disc displacement by USI, relative to MRI, showed a $100 \% \mathrm{Se}, 82.76 \% \mathrm{Sp}$, 93.15\% PPV, 100\% NPV, and 94.85\% accuracy, while the detection of joint effusion by USI showed a Se, Sp, PPV, NPV, and accuracy of $100 \%$. USI indicated strong agreement with CBCT, which showed a $98.08 \%$ Se, $94 \%$ Sp, $94.44 \%$ PPV, 97.92\% NPV and 96.08\% accuracy for detecting condylar irregularities. In contrast, MRI showed an Se of $100 \%$, Sp of $56.86 \%$, PPV of $69,86 \%$, NPV of $100 \%$, and accuracy of $78.43 \%$ for detecting condylar irregularities, relative to $\mathrm{CBCT}$.

\section{Discussion}

In this study, high-resolution ultrasound was an accurate method to differentiate between normal and displaced disc positions. Additionally, we distinguished between the articular disc and capsule, between the two hyperechogenic lines that are related to the cortex condyle head and the cortex of the articular eminence, and between the articular disc that appeared as a hypoechoic area between the lines; these results are an agreement with those of another study $(6,10,13)$. Per Kalyan et al., Lands et al. stressed that visualization of the articular disc in all cases is impossible and that imaging protocols not standardized because the definitions and echogenic properties of the disc are not the same in different studies (14). The articular disc was first visualized by ultrasonography in 1991 and 1992 by Nabieh and Speculand, with a $3.5 \mathrm{MHz}$ transducer $(15,16)$

In the comparison between USI and MRI, USI demonstrated a high Se, Sp, PPV, NPV and accuracy in the detection of ADD, joint effusion, and condylar irregularities. Many investigations have discussed the efficacy of USI as a diagnostic method for detecting ADD (6). The results from Razek et al. (6) revealed that ultrasound had a Se of 79.3\%, Sp of $72.7 \%$, and PPV and NPV of $88.5 \%$ and $57.1 \%$, respectively (6). However, a study conducted by Yang et al. (17) showed that USI had a Se, Sp, accuracy, PPV, and NPV of $82.6 \%, 94.1 \%, 92.5 \%, 95 \%$, and $80 \%$, respectively. Therefore, this study agrees with the results obtained in previous research and shows that USI is an effective method for diagnosing TMJ pathologies. The imaging parameter values in this study are much higher than those in previous studies, which could be related to the practitioner skills and the high-frequency ultrasound probe $(18 \mathrm{MHz})$; these higher values lead to more detailed images and improvements in soft tissue differentiation, which translates to advancements in image quality and better diagnoses. The study conducted by Jank et al. (18) found that not all disc displacements that were detected and diagnosed via USI could be identified by MRI . However, some of the authors consider MRI as the gold standard imaging technique for detecting TMDs (4).

In contrast, according to Bas et al. (5), the only scenario in which imaging is needed for TMDs is when imaging could provide critical information that would influence treatment decisions. Currently, MRI is not available in most TMD clinics; furthermore, MRIs are expensive examinations because of equipment costs, facilities costs, staffing and availability, all of which limit the use of this imaging modality in many countries. Additionally, MRI 
is contraindicated in some individuals, such as those with pacemakers (however, new pacemaker materials do not interfere with MRI examinations) (19), intracranial vascular clips and metal particles in the eye or other vital structures (5). Other contraindications include obesity and claustrophobia (5) due to the small diameter of the magnet bore.

Moreover, MRI examinations are carried out with the head of the patient placed in an abnormal head position, which can affect mandibular motion (14). Of the 102 patients included in this study, 77 were female, and 25 were male. Statistical analysis showed that there is a significant association between sex and TMD. This male-to-female ratio supports findings from other studies that indicate that female hormones play a significant role in the pathogenesis of TMDs $(16,18,20)$.

On ultrasound, the articular disc appears as a hyperechogenic thin area surrounded by a hypoechoic area. In this study, a normal disc position was described as when the anterior border of the disc was located superior to the condyle (Figure 2), and the disc considered was anteriorly displaced when the anterior border of the disc was presented anterior to the condyle. Effusion was identified when the articular disc width was $2 \mathrm{~mm}$ or higher and the hypoechoic area was within the thin articular space $(21,22)$. In the chronic inflammatory process, the capsule was observed to be fibrosed, thus exhibiting a higher echogenicity and a fibrosed disc (23).

Most patients diagnosed with joint effusion and condylar irregularities in this study showed a high correlation with TMJ pain through extraoral examinations. These results agree with a survey carried out by Thomas et al. (16), which showed a significant strong correlation between joint effusion and TMJ pain. Additionally, that study reported a strong correlation between the presence of a clicking noise and surface irregularities (16). The study performed by Katzberg et al. (24) mentioned that effusion is caused by an increase in the volume of the inflammatory exudate and can activate the nociceptive neurons in the joint and trigger pain . In this study, the Se, Sp, PPV, NPV, and accuracy of USI for detecting joint effusion was $100 \%$ relative to MRI. These values are higher than those in a 2006 study by Jank et al. (18); in that study, USI had a Se of $81 \%$, Sp of $100 \%$, and accuracy of $95 \%$ for detecting joint effusion, which means that USI is a reliable diagnostic method for detecting joint effusion. According to a study by Manfredini et al. (25), USI shows an acceptable accuracy for the diagnosis of effusion.
In the present study, the detection of joint effusion by USI was precisely accurate, and the results obtained from USI coincide with those from MRI.

On the USI scans and during movements to open and close the mouth, the articular disc is quickly recognized. ADDwor was identified when the disc was positioned in the anterior aspect of the condyle both while the mouth was in the closed and in the maximum opening positions. In contrast, ADDwr was identified when the disc was positioned anterior to the condyle when the mouth was closed but returned to the normal position when the mouth was opened. Many studies indicate that the visualization of ADDwr is difficult with USI because soft tissue and liquids are better detected than mineralized tissue (24). Another study proposed that the difficulty in detecting ADDwr is due to the suboptimal depiction of articulation components, such as the disc, glenoid fossa and condyle (4). This paper disagrees with the results of previous studies because in the present study, USI was efficient in visualizing ADD with reduction; ADD was mainly seen during dynamic exploration on the sonogram. However, a study by Hayashi et al. (26) with more than 23 patients and a $10 \mathrm{MHz}$ transducer reported a Se of $63 \%$, Sp of $100 \%$ and accuracy of $72 \%$ for detecting disc displacement, while Manfredini et al. (20) reported a Se and Sp of $57 \%$ and $74 \%$ for detecting disc displacement, respectively. Kaya et al. (27) stated that there were no significant differences between MRI and USI in visualizing ADD, ADDwr, ADDwor, and effusion, and the findings of the two methods agreed with each other in all assessments $(p>0.05)$. Dong et al. (4) reported that patients who were suspected to have ADDwr were associated with an $89 \%$ probability of having a positive ADDwr result and a $21 \%$ chance of having a negative result after being evaluated by USI. In comparison, the corresponding probabilities for ADDwor were $82 \%$ and $16 \%$, respectively (4). In the present study, USI demonstrated a high Se, Sp, PPV, and NPV for detecting ADD. In this study, there were only four patients with ADDwor, which could be related to the patient cohort. However, the results of USI and MRI for ADDwor were in an agreement. We have found that ultrasound is a potential diagnostic method to detect TMD pathologies, and we performed comparisons between the two diagnostics methods utilized in this study. Additionally, this study correctly distinguished between ADDwr and ADDwor and thus, we disagree with the study from Emshoff et al. (28) that reported that USI is not useful in distinguishing between disc displacement with and without reduction. 
Emshoff et al. (11) found that USI is an inadequate imaging technique for detecting condylar irregularities; however, this is not true because in the present study, USI was useful and accurate for detecting condyle irregularities and morphological abnormalities on the cortical surface of the condyle. Figure 6 shows representative scans of the same patient with the two imaging modalities, and these images show a diagnostic agreement between USI and CBCT in terms of the subchondral bony osteophyte on the right condyle.
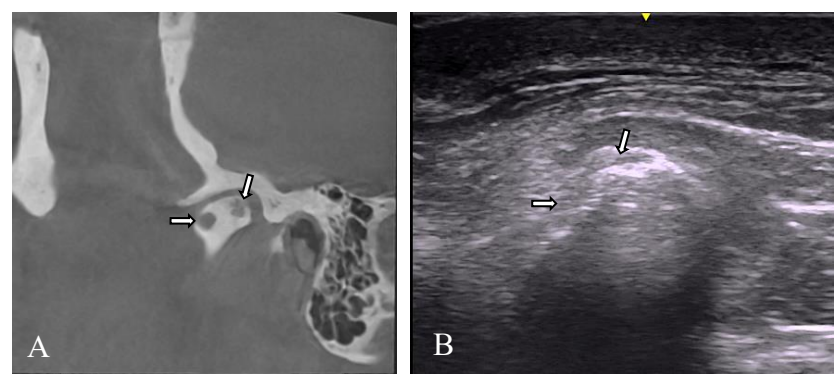

Figure 6. A. CBCT of the right mandibular condyle showing a subchondral bony osteophyte indicated by the arrows. B. USI of the right condyle showing a hypoechoic area, indicated by the arrows, which is compatible with a subchondral bony osteophyte.

In the comparison between USI, MRI, and CBCT for detecting condylar irregularities, $\mathrm{CBCT}$ confirmed the presence of bone irregularities in 51 patients, who were previously diagnosed by USI. In contrast, MRI showed low efficacy in the detection of bone abnormalities on the cortical surface of the condyle; this result is in agreement with the Alkhader et al. (29) study that reported that MRI showed a relatively low Se (30-82\%) for detecting bony abnormalities of the TMJ . The value of MRI for detecting TMJ bony defects is considered to be limited (29). This finding is coincident with the results of the present study, in which MRI showed a high Se but relatively low Sp for detecting condylar irregularities in comparison with other imaging modalities; USI and CBCT had similar results concerning the detection of hard tissue abnormalities. Most of the previous publications were concerned with the issue of disc displacement and TMD pathologies. However, it is essential to remember that USI is not limited to only diagnosing ADD but also can detect joint effusion, joint degeneration, and condylar irregularities. Additionally, USI can be used for muscular examinations, as in the study by Sasaki et al. (30), where USI was used to clarify changes in the master muscle after splint therapy. A limitation of this study is visualization of the medial pole of the condyle, which cannot be seen on USI. Therefore, the articular disc position cannot be evaluated in mediolaterally. This paper achieves the recommendation of the previous study by Borahan et al. (2) published in 2018, which states that a large sample is necessary to assess the Se and Sp of USI relative to MRI. From this study, we can conclude that USI is a useful diagnostic method with a high Se, Sp, PPV and NPV and that these results are consistent with other studies that demonstrate the efficacy of USI in diagnosing TMDs, as well as its potential to be used as an alternative imaging technique to monitor the TMJ $(23,31)$. USI has many advantages over other imaging modalities, including its low cost as an imaging method, ease of manipulation by experienced examiners, and its non-invasive nature that eliminates the radiation risk for patients. USI can also be useful in detecting lateral excursion mandibular movements (5). Overall, this imaging modality allows us to visualize the real-time static and dynamic movements of the condyle and its association with the articular disc. Another great advantage of this imaging modality is the possibility for repeated examinations with no radiation exposure, which is not possible with other imaging techniques. Future investigations with high-resolution 3D ultrasonography are necessary to assess the medial pole of the condyle, which could provide an additional advantage towards USI being considered the gold standard diagnostic method.

\section{Conclusion}

High-resolution ultrasonography is a useful diagnostic method; USI can supplement clinical evaluations in patients with TMDs and can be used as a diagnostic tool to identify ID of the TMJ. USI is a non-invasive and inexpensive diagnostic method that is easy to use with no radiation exposure, and this imaging modality can be used in routine clinical practice to evaluate TMDs. Due to its high Se, Sp, PPV, NPV, and accuracy for detecting ADD, joint effusion and condylar irregularities, ultrasonography can be suggested as an alternative imaging method for TMJ assessments. Future studies with 3D ultrasonography are required to assess the medial pole of the condyle.

\section{References}

1. Murphy MK, MacBarb RF, Wong ME, Athanasiou KA. Temporomandibular joint disorders: A review of etiology, clinical management, and tissue engineering strategies. The 
International journal of oral \& maxillofacial implants. 2013 Nov;28(6):e393.

2. Keser G, Ulay G, Namdar Pekiner F, Borahan MO. Evaluation of Diagnostic Efficiency of Ultrasonography in Temporomandibular Joint Disorders: A Pilot Study. Marmara Dent J. 2018;2(1):7-12.

3. Li C, Su N, Yang X, Yang X, Shi Z, Li L. Ultrasonography for detection of disc displacement of temporomandibular joint: A systematic review and meta-analysis. J Oral Maxillofac Surg. 2012;70(6):1300-9.

4. Dong XY, He S, Zhu L, Dong TY, Pan SS, Tang LJ, et al. The diagnostic value of high-resolution ultrasonography for the detection of anterior disc displacement of the temporomandibular joint: A meta-analysis employing the HSROC statistical model. Int J Oral Maxillofac Surg. 2015;44(7):852-8.

5. Bas B, Ylmaz N, Gkce E, Akan H. Diagnostic value of ultrasonography in temporomandibular disorders. J Oral Maxillofac Surg . 2011;69(5):1304-10.

6. Razek AAKA, Al Mahdy Al Belasy F, Ahmed WMS, Haggag MA. Assessment of articular disc displacement of temporomandibular joint with ultrasound. J Ultrasound . 2015;18(2):159-63.

7. Kundu H, Basavaraj P, Kote S, Singla A, Singh S. Assessment of TMJ disorders using ultrasonography as a diagnostic tool: A review. J Clin Diagnostic Res. 2013;7(12):3116-20.

8. Osiewicz MA, Lobbezoo F, Loster BW, Loster JE, Manfredini D. Frequency of temporomandibular disorders diagnoses based on RDC/TMD in a Polish patient population. Cranio J Craniomandib Pract . 2018;36(5):304-10.

9. Klatkiewicz T, Gawriołek K, Pobudek Radzikowska M, Czajka-Jakubowska A. Ultrasonography in the Diagnosis of Temporomandibular Disorders: A Meta-Analysis. Med Sci Monit . 2018;24:812-7.

10. Emshoff R, Jank S, Rudisch A, Walch C, Bodner G. Error patterns and observer variations in the high-resolution ultrasonography imaging evaluation of the disk position of the temporomandibular joint. Oral Surg Oral Med Oral Pathol Oral Radiol Endod. 2002;93(3):369-75.

11. Emshoff R, Brandlmaier I, Bodner G, Rudisch A. Condylar erosion and disc displacement: Detection with high-resolution ultrasonography. J Oral Maxillofac Surg. 2003;61(8):87781.

12. Emshoff R, Bertram S, Rudisch A, Gaßner R. The diagnostic value of ultrasonography to determine the temporomandibular joint disk position. Oral Surg Oral Med Oral Pathol Oral Radiol Endod. 1997;84(6):688-96.

13. Emshoff R, Jank S, Bertram S, Rudisch A, Bodner G. Disk displacement of the temporomandibular joint: Sonography versus MR imaging. Am J Roentgenol. 2002;178(6):155762.

14. Kalyan US, Moturi K, Rayalu KP. The Role of Ultrasound in Diagnosis of Temporomandibular Joint Disc Displacement : A Case - Control Study. J Maxillofac Oral Surg . 2017

15. Nabeih YB, Speculand B. Ultrasonography as a diagnostic aid in temporomandibular joint dysfunction. Int $\mathrm{J}$ Oral Maxillofac Surg . 1991;20(3):182-6.
16. Thomas AE, Kurup S, Kumar SP, Chandy ML, Jose R. Diagnostic efficiency of high-resolution ultrasonography in patients with chronic temporomandibular disorders. Oral Radiol. 2016;32(3):160-6.

17. Yang J, Liu W, Zhong Y, Zhao H. The diagnostic value of high-resolution ultrasonography for detecting anterior disc displacement without reduction of temporomandibular joint. Hua xi kou qiang yi xue za zhi= Huaxi kouqiang yixue zazhi=West China journal of stomatology. 2012 Dec;30(6):632-4.

18. Jank S, Emshoff R, Norer B, Missmann M, Nicasi A, Strobl $\mathrm{H}$, et al. Diagnostic quality of dynamic high-resolution ultrasonography of the TMJ - A pilot study. Int J Oral Maxillofac Surg . 2005;34(2):132-7.

19. Markman TM, Halperin HR, Nazarian S. Update on MRI Safety in Patients with Cardiac Implantable Electronic Devices. Radiology. 2018;288(3):656-7.

20. Manfredini D, Tognini F, Melchiorre D, Zampa V, Bosco M. Ultrasound assessment of increased capsular width as a predictor of temporomandibular joint effusion. Dentomaxillofacial Radiol. 2003;32(6):359-64.

21. Jank S, Rudisch A, Bodner G, Brandlmaier I, Gerhard S, Emshoff R. High-resolution ultrasonography of the TMJ: Helpful diagnostic approach for patients with TMJ disorders? J Cranio-Maxillofacial Surg. 2001;29(6):366-71.

22. Liao LJ, Lo WC. High-Resolution Sonographic Measurement of Normal Temporomandibular Joint and Masseter Muscle. J Med Ultrasound . 2012;20(2):96-100.

23. Landes C, Walendzik H, Klein C. Sonography of the temporomandibular joint from 60 examinations and comparison with MRI and axiography. J Cranio-Maxillofacial Surg. 2000;28(6):352-61.

24. Katzberg RW. Is ultrasonography of the temporomandibular joint ready for prime time? Is there a "window" of opportunity? J Oral Maxillofac Surg . 2012;70(6):1310-4.

25. Manfredini D, Guarda-Nardini L. Ultrasonography of the temporomandibular joint: a literature review. Int $\mathrm{J}$ Oral Maxillofac Surg. 2009;38(12):1229-36.

26. Hayashi T, Ito J, Koyama J, Yamada K. The accuracy of sonography for evaluation of internal derangement of the temporomandibular joint in asymptomatic elementary school children: comparison with MR and CT. AJNR Am J Neuroradiol . 2001;22(4):728-34.

27. Kaya K, Dulgeroglu D, Unsal-Delialioglu S, Babadag M, Tacal T, Barlak A, et al. Diagnostic value of ultrasonography in the evaluation of the temporomandibular joint anterior disc displacement. J Cranio-Maxillofacial Surg . 2010;38(5):3915.

28. Emshoff R, Jank S, Bertram S, Rudisch A, Bodner G. Disk Displacement of the Temporomandibular Joint Sonography Versus MR Imaging American Journal of Roentgenology Vol. 2002; (June 2000):1557-62.

29. Alkhader M, Ohbayashi N, Tetsumura A, Nakamura S, Okochi $\mathrm{K}$, Momin MA, et al. Diagnostic performance of magnetic resonance imaging for detecting osseous abnormalities of the temporomandibular joint and its correlation with cone beam computed tomography. Dentomaxillofacial Radiol. 2010;39(5):270-6. 
30. Sasaki J, Ariji Y, Sakuma S, Katsuno R, Kurita K, Ogi N, et al. Ultrasonography as a tool for evaluating treatment of the masseter muscle in temporomandibular disorder patients with myofascial pain. Oral Radiol. 2006;22(2):52-7.
31. Y1lmaz D, Kamburoğlu K. Comparison of the effectiveness of high resolution ultrasound with MRI in patients with temporomandibular joint disorders. Dentomaxillofacial Radiol. 2019;20180349. 